\title{
To Die or Not To Die? Current Questions in Cell Death Signalling
}

\author{
H. Anne Leaver • Giles Hardingham
}

Published online: 18 May 2010

(C) Springer Science+Business Media, LLC 2010

A philosopher once stated that 'over every good laboratory, there hangs a question mark' [George MacLeod 1939, The Coracle], and it is certainly the case that questions surrounding the mechanism and role of cell death have hung over many laboratories over recent years. Cell death signalling has provided a fruitful area of enquiry, and it is now possible to carry out functional analysis of linked systems of signals, receptors, membranes, organelles and mediators involved in cell death signalling. But in such analysis, a central question, discussed in this edition of Molecular Neurobiology, relates to decision points: Under which circumstances and at what point do cells commit to die? This is an important question, not least because, in cases of pathological cell loss or gain, it marks the point after which therapeutic intervention may be futile, while, during development, it marks the point at which external cues are integrated to instruct a cell to live or to die.

The diverse cell death signalling pathways leading to these decision points are discussed [Wyllie; Lukiw and Bazan] including oxidative and non-oxidative signalling pathways, inflammatory and non-inflammatory signals; responses of differently dividing and differentiated cell types; and sensitivity to environmental and therapeutic conditions. Further, the key molecular determinants of this decision involve both a lack of trophic stimuli [Brady and Morfini] as well as the active effects of co-ordinated cell

\footnotetext{
H. A. Leaver $(\triangle)$

Department of Clinical Neurosciences, University of Edinburgh, Edinburgh, UK

e-mail: hanne.leaver@googlemail.com

G. Hardingham

Centre for Neuroscience Research, University of Edinburgh, Edinburgh, UK

e-mail: Giles.Hardingham@ed.ac.uk
}

death signals [Colquhoun]. Key effectors and integrators of the decision process, the intrinsic and extrinsic apoptotic signalling pathways, are discussed by Wyllie, Whittle, Rizzo, Colqhoun and Leaver. Andrew Wyllie describes the diversity of early signals and events leading to cell death, and how cross-talk between sub-lethal signals may cumulatively tip a cell or system towards cell death. He proposes that signalling processes involved in normal physiological functions can become involved in death under pathological conditions.

Various aspects of brain-specific microenvironments are considered in this series of Cell Death Signalling reviews. The unique properties of brain endothelial cells and their response to stress signalling are described by Maria Teresa Rizzo. Brain endothelial cells may exacerbate, or even initiate, inflammatory, ischemic and degenerative disorders of the central nervous system. Both acute and chronic signals which activate endothelial cell death signalling are discussed. Important responses include stress kinase activation and changes in gene expression, but these responses depend on cellular contexts such as differentiation status and microenvironment. Rizzo proposes that the limited success of recent neuroprotective therapies relate to failure to consider the integrated response of the neurovascular unit. Glioma mitochondrial metabolism is discussed by Whittle et al., including mitochondrial-specific ketone metabolism in the Warburg hypothesis, and recent evidence for Akt oncogene expression and associated anaerobic glycolysis in malignant gliomas. Also, abnormalities in cardiolipin structure and distribution in glioma mitochondria are associated with disorders of mitochondrial respiratory complex function. Proteomic analysis of gliomas has revealed abnormalities in the expression of key components of the intrinsic apoptosis pathway. Further support for specific mitochondrial changes leading to cell death in 
glioma is given by Colquhoun, including changes in the activity of carnitine palmitoyl transferase 1 , interactions of hexokinase with the mitochondrial outer membrane voltagedependent anion channel and stress kinase (Akt) activation. The interaction of these signals with radiotherapy and chemotherapy may increase tumour sensitivity and improve tumour responses to conventional treatment, which is currently a major problem in glioma therapy.

In contrast, the neurodegenerative disease spectrum exposes different aspects of cell death signalling pathology, in which inflammatory signalling may be harmful, rather than useful in therapeutic strategy. The role of pro and antiinflammatory signalling in neurodegenerative disease is discussed by Nicolas Bazan. Inflammatory signalling involving cytosolic phospholipase $\mathrm{A}_{2}$ and changes in proand anti-apoptotic members of the Bcl-2 gene family are analysed in terms of stress signalling involving NF-kappa B-regulated genes and age-related pathologies. A further signal for degenerative processes in the cornea is discussed by Haydee Bazan. The mediator, platelet-activating factor (PAF) produced by many cells and tissues in response to injury, has vasoactive and pro-inflammatory actions in the cornea, activating inflammatory cells including neutrophils, eosinophils and macrophages and acting as an early mediator of injury and stromal cell apoptosis. As a result, PAF antagonists have potential in limiting corneal injury [Esquenazi and Bazan].

Considering the frequent references in the cell death signalling literature to inflammatory processes, mediators and cells, it is surprising how little is known about initiating events in inflammatory cell recognition, both in the periphery and in the brain [Zhang, Shipston and Brown]. It is salient that potassium leak represents an early feature of cells destined to die by apoptosis that could trigger depolarization of phagocytic cells nearby. Thus, cells in the early stages of apoptosis may use potassium ion flux to modulate the inflammatory profile of responding phagocytes, influencing their cytokine release, signalling and function. This is an important new hypothesis, linking ion flux in apoptotic cells and the reticuloendothelial system with the control of inflammation.

A characteristic of neuronal tissue is its lipid composition, including high polyunsaturated fatty acid and omega-3 content, and this signalling is analysed in both neurodegenerative and oncogenic processes in this special edition. Nicolas Bazan relates omega-3 metabolism to inflammatory signalling in Alzheimer's disease and Gene Anderson and Haydee Bazan analyse specific aspects of polyunsaturated fatty acid metabolism in the context of retinal and corneal function. Michel Lagarde discusses the selective brain uptake of specific omega-3 fatty acids, and lipid metabolism is also discussed by Rizzo, Colquhoun and Leaver in the context of endothelial and glial cell death signal transduction. Recent advances defining the role of docosahexaenoic acid (DHA) in neuronal, synaptic and retinal membranes, and the anti-inflammatory properties of the DHA metabolite neuroprotectin D1 are highlighted by Nicolas Bazan, while the uptake of DHA into the brain by specific esterification/re-esterification pathways is analysed by Lagarde. Both site specific and general mechanisms of apoptosis lipid signalling are also discussed by Eugene Anderson. Novel aspects of phosphoinositide signalling in retinal function are described [Rajala and Anderson]. Photoactivation of rhodopsin activates phosphorylation of the insulin receptor, leading to Akt activation, which promotes neuronal survival by inactivating components of the apoptotic machinery. Thus, rhodopsin is involved in cross-talk with signalling pathways beyond the phototransduction cascade, feeding into the Akt pathway common to cell death signalling in other lipid signalling systems.

Special conditions leading to neurodegenerative cell death are discussed by Scott Brady, who highlights the importance of structural and spatiotemporal organisation in neuronal pathology, and argues that axonal transport is a crucial factor in cell death decisions. Indeed, Brady suggests that previous work, focussing primarily on cell death signals that occur late in neurodegeneration, rather than on earlier changes in neuronal function and connectivity, has distorted therapeutic studies and delayed advances in this area. The synaptic and axonal activity necessary for maintaining function may lead to pruning of unnecessary links during development, but may be exaggerated in neurodegenerative disease, shutting down presynaptic termini, leading to dying back neuropathy in distal axons. In Alzheimer's disease, hippocampal and pyramidal neurones of the association neocortex show loss of arborisation and increased inflammatory, stress and pro-apoptotic signals [Lukiw and Bazan]. Structural and spatiotemporal organisation is also becoming increasingly relevant in neoplastic studies of co-ordinated glioma cell death involving glial-endothelial cell signalling [Leaver, Rizzo and Whittle].

On a practical level, a novel approach to neuronal signalling research using human embryonic stem cells is described by Giles Hardingham. Human embryonic stem cells provide a complementary model to rodent studies and the relatively small amounts of human ex vivo tissue available for studying neuronal function and degenerative processes. The application of human neuronal stem cells to studies of oxidative and glutamate induced stress may overcome certain issues of species-specific differences, in areas such as gene expression and receptor pharmacology. Stem cell models are also useful in studying glioma signalling and the development of glioma [Leaver, Rizzo and Whittle]. Stem cell analysis may also increase our understanding of signals associated with glioma cell death. 
Polyunsaturated fatty acids and stress signals exert inhibitory effects on tumour proliferation, inhibit angiogenesis, expression of proteins affecting endothelial gap junctions and endothelial tube formation [Colquhoun].

In conclusion, new approaches to cell death signalling, involving study of individual neuronal systems, their microenvironment and cellular interactions, are necessary, now that many mediators and effector pathways of cell death have been identified. Ion flux, neurotransmission and mitochondrial metabolism all provide crucial information about initial signalling, pro-survival and pro-apoptotic signal transduction and the crucial decision points in cell death in neurodegenerative and oncogenic pathways.
Acknowledgements We are very grateful to Dino Rotondo [University of Strathclyde] for invaluable help in organising the programme and special edition, to Jill Davidson [University of Strathclyde] and Peng Lee Yap [Clinical Immunology, Edinburgh] for skilful editorial and scientific input, to Nicolas Bazan [University of New Orleans] and John Kelly [University of Edinburgh] for oversight and encouragement, to young scientists Svitlana Loughridge [Cell Biology R\&D, Edinburgh] and Sarah Bott [Cell Biology R\&D, Edinburgh], for practical support, to Ian Whittle and Ian McCutcheon [University of Edinburgh] and to the Edinburgh Conference Bureau for organisational assistance, and to Amarin, Antoxis, Enzo Life Sciences, Promega, and the Royal Society of Edinburgh for the financial support which made the meeting possible.

H Anne Leaver and Giles Hardingham January 2010 